\title{
Automated quality control for the haematology laboratory
}

\author{
I. CAVILL AND C. RICKETTS
}

From the Department of Haematology, University Hospital of Wales, Cardiff

SYNOPSIS Two simple methods of using sophisticated statistical techniques to analyse intralaboratory quality control data are described. One method uses a plastic grid to assess the cumulative sum (cusum) graph. An equivalent procedure, which may be carried out by a laboratory computer, gives automated on-line quality control of the Coulter S blood counter. The possible development of simple apparatus to carry out the same function is discussed.

Quality control in the laboratory is carried out by including control samples in the routine procedure and monitoring the level of the relevant constituent. The most sensitive and effective way of analysing the control data is by means of the cumulative sum (cusum) technique. A system of intralaboratory quality control has been described together with a simple visual method for evaluating control results (Cavill and Jacobs, 1973). In a large and busy laboratory there is a need for a rapid method of assessing the cusum data-preferably one which can be automated or carried out by a laboratory computer. The present paper shows how a sophisticated mathematical test for loss of control can be applied in the laboratory using a transparent plastic grid on which the limits of acceptability have been marked and gives details of how this method can be applied using a small laboratory computer interfaced on-line with a Coulter S. The possible development of a simple apparatus for automated intralaboratory quality control is discussed.

\section{The Cusum Technique}

The cusum is the cumulative sum of the differences between each control value and a reference value. The graph of cusum against control number is a sensitive reflection of the accuracy of the control data. As long as the mean value of the control is equal to the reference value the path of the graph will fluctuate about a horizontal line. If the mean changes the direction of the path will change. The frequency with which the difference exceeds zero increases when the mean of the control increases to a new value and accordingly the path of the graph Received for publication 23 May 1974. slopes upwards. Conversely it will slope downwards if the mean control level drops below the reference value. A sustained change in direction of the path is taken as an indication of a change in the mean level of the control. Experience has shown that when the distance between successive points on the horizontal axis is equal to $\nu$.SD (standard deviation) on the vertical axis then optimum sensitivity is obtained when $\nu=2$. In practice $\nu$ is kept as close to 2 as possible while keeping the vertical scale in easily plotted units.

Although the calculations and plotting involved in a cusum analysis are simple they are nevertheless time-consuming. In a busy haematology laboratory this may be a significant factor in limiting throughput. A simple geometric device taking the form of a $V$ marked on a piece of perspex can be used to provide a rapid and exact assessment of the cusum (Barnard, 1959). The two limbs of the $V$ are inclined at an angle $\theta$ to the line OP of length $\mathrm{d}$. The $\mathrm{V}$ mask is used by placing it on the chart so that the line OP is horizontal and point $\mathbf{P}$ coincides with the last plotted point (fig 1). If the path joining the points of the graph crosses either limb of the $V$ the mean value of the control has changed.

\section{Calculation of $\mathbf{d}$ and $\theta$}

The control procedure should indicate significant changes in the control mean quickly but should give only a low rate of false positive warnings. The mean number of control samples tested before a change in the control mean is detected is called the average run length (ARL).

A suitable V-mask will give an ARL in the region of 500 samples when testing is in control and of 


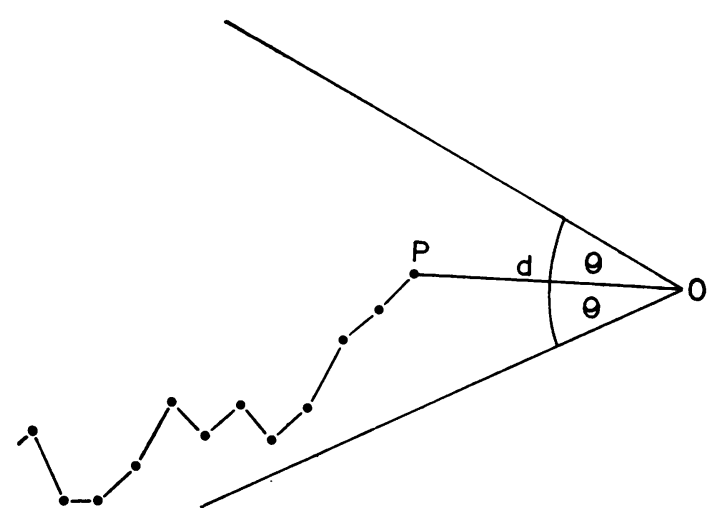

Fig 1 The V-mask is placed on the cusum chart with the line OP horizontal and the point $P$ coinciding with the last plotted point. Each arm of the $V$ is inclined at an angle $\theta$ to $O P$ which is of length $d$.

three samples when the control mean changes by 2 standard deviations. Goldsmith and Whitfield (1961) have shown that a V-mask with $\tan \theta=\frac{1}{v}$ and $d=2.6$ will satisfy these criteria.

\section{EXAMPLE}

The haemoglobin concentration from control samples inserted into the routine procedure after every 20 tests is shown in figure 2 . The mean for this control was $9.6 \mathrm{~g} / \mathrm{dl}$ and the standard deviation for the test was $0.1 \mathrm{~g} / \mathrm{dl}$. In the diagram $\nu=2$, thus $\theta=27^{\circ}$. The V-mask indicates a change in the mean level of the control at the eighteenth sample.

\section{AUTOMATED QUALITY CONTROL}

When a Coulter S (Coulter Electronics Ltd, Dunstable, Beds) or any other instrument is interfaced

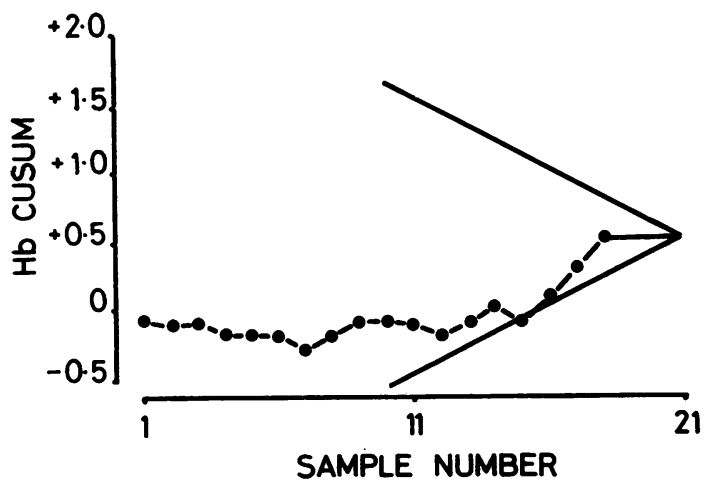

Fig $2 A V$-mask with $d=2.6$ and $\theta=27^{\circ}$ detects $a$ change in the mean haemoglobin concentration at the eighteenth control sample. on-line to a laboratory computer it is possible to automate the analysis of the control results. The computer performs a series of calculations which are exactly equivalent to the $V$-mask analysis on each of the four parameters measured directly by the Coulter S: white cell count (WBC), red cell count (RBC), haemoglobin concentration (Hb), and mean corpuscular volume (MCV). The complete analysis of the four results for each control sample takes less than one second, after which an appropriate message is printed on a teletype near the Coulter S. In our laboratory the Coulter $S$ is linked to a PDP8/e computer (Digital Equipment Co, Reading) with 8K core and a 32K fixed head disc (Cavill, Ricketts, Moulding, Jacobs, and Page, 1974). The decisions and calculations carried out by the computer are very simple although with a floating point arithmetical package the whole quality control program occupies about $2 \mathrm{~K}$ of core.

The mean value for each parameter is calculated from 10 measurements on each new control batch. Two reference values, upper(URV) and lower (LRV), are calculated from the control mean and the SD for the test using the formulae URV $=\mu+k$ and $\mathbf{L R V}=\mu-\mathbf{k}$, where $\mu$ is the control mean and $k$ is given by $\mathrm{k}=2 \mathrm{SD} \cdot \tan \theta$.

As long as the subsequent control values lie between these two reference values no further calcu lations are made. When these limits are exceeded cusum calculation about the appropriate reference value is automatically started. If this cusum exceeds a predetermined value, the decision interval, the parameter is out of control. A message is then printed on a teletype adjacent to the Coulter $S$, the cusum is reset to zero and the machine must be restandardized. The decision interval, $h$, is given by $\mathrm{h}=2 \mathrm{SD} \mathrm{d} \tan \theta$. If, during a cusum calculation, the cusum returns to zero or changes sign, then the cusum is automatically reset to zero. The computer procedure is shown in the flow charts in figure 3.

\section{OPERATION}

1 The Coulter should be calibrated precisely to the 4C mean reference values.

2 The mean value of the control preparation should be calculated from 10 replicate measurements.

3 Each control sample is preceded by a wash. The control is identified to the computer using a small data entry terminal beside the Coulter $S$.

4 Reference values in this laboratory are set at the following values from the mean: WBC \pm 0.3 $\times 10^{9} / 1 ; \mathrm{RBC} \pm 0.05 \times 10^{12} / \mathrm{l} ; \mathrm{Hb} \pm 0.1 \mathrm{~g} / \mathrm{dl}$; $\mathrm{MCV} \pm 1 \mathrm{fl}$.

When the mean lies between two Coulter values it 

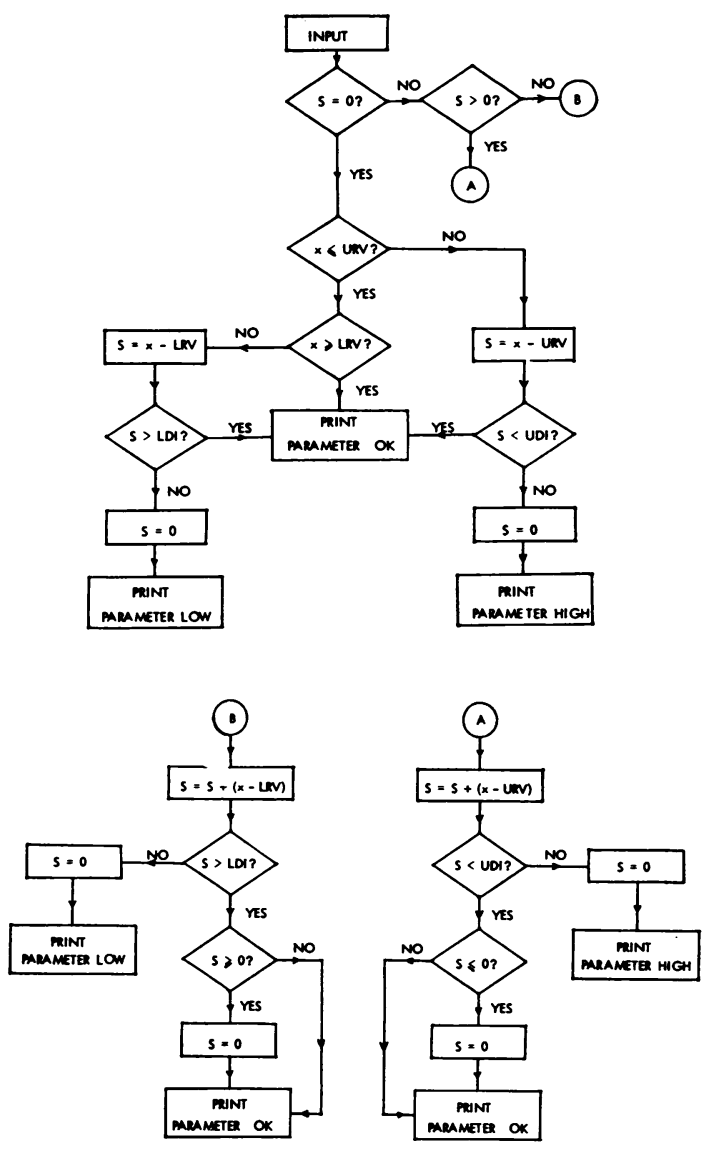

Fig 3 The computer procedures for detecting a change in the control mean are shown in the flow charts. $S$ represents the cusum and $x$ the current control result. $L R V$ and $U R V$ are the lower and upper reference values. The lower and upper decision intervals, LDI and $U D I$, are set at $-h$ and $+h$ respectively.

is rounded up to calculate the upper reference value and rounded down for the lower reference value.

The decision intervals for each parameter are: $\mathrm{WBC} \pm 0.8 \times 10^{9} / 1 ; \mathrm{RBC} \pm 0.13 \times 10^{12} / 1 ; \mathrm{Hb} \pm$ $0.3 \mathrm{~g} / \mathrm{dl} ; \mathrm{MCV} \pm 3 \mathrm{fl}$.

5 If the quality control for any parameter is not acceptable then the machine is recalibrated as in (1).

\section{Discussion}

A computerized quality control system has been in operation in the laboratory at the University Hospital of Wales for the past year. The rapid and unequivocal evaluation of control data has relieved the laboratory staff of a considerable amount of tedious but exacting work while still maintaining active quality control with up to 500 samples analysed by the Coulter each day. Moreover, the success of this system has allowed us to issue Coulter $\mathbf{S}$ reports directly from the laboratory without the need for further checking.

In order to make full use of this system and to control all four parameters measured by the Coulter $\mathrm{S}$, we have used a commercial control preparation. This is produced in bulk and supplied unassayed by Travenol Laboratories (Thetford, UK) at monthly intervals. The red and white cell counts and the haemoglobin concentration are completely stable but the MCV shows considerable variation which appears to be temperature dependent. We allow the control preparation to equilibrate at room temperature for three hours after it is removed from storage at $0-4^{\circ} \mathrm{C}$. In addition, the inflowing Coulter diluent is passed through a coil immersed in a $37^{\circ} \mathrm{C}$ waterbath. These two measures have produced a satisfactorily stable MCV for the control preparation.

Although we have used a laboratory computer to automate the cusum analysis in the laboratory this could probably be carried out on a much simpler computer or even on an electronic calculator. If such an instrument could be interfaced on-line toa Coulter counter or to any other automated analyzer, and could give a simple indication of whether or not the apparatus is in control then the same quality control system could be applied. A simple 'black box' attached directly to the Coulter and carrying out these specific procedures might be one possible solution. This would be much cheaper but just as effective as the computerized system which we have developed.

\section{References}

Barnard, G. A. (1959). Control charts and stochastic processes. J. roy. statist. Soc. Ser. B, 21, 239-271.

Cavill, I., and Jacobs, A. (1973). Quality control in haematology Association of Clinical Pathologists: Broadsheet 75.

Cavill, I., Ricketts, C., Moulding, T., Jacobs, A., and Page, M. (1974). A system for data processing in haematology. J. clin. Path., 27, 330-333.

Goldsmith, P. L., and Whitfield, H. (1961). Average run lengths in cumulative chart quality control schemes. Technometrics, 3, 11-20. 